\title{
Simulating Dynamic Time Dilation in Relativistic Virtual Environment
}

\author{
Abbas Saliimi Lokman, Ngahzaifa Ab. Ghani, and Lok Leh Leong
}

\begin{abstract}
This paper critically reviews several Special Relativity simulation applications and proposes a method to dynamically simulate Time Dilation effect. To the authors' knowledge, this has not yet been studied by other researchers. Dynamic in the context of this paper is defined by the ability to persistently simulate Time Dilation effect based on specific parameter that can be controlled by user/viewer in real-time interactive simulation. Said parameter is the value of speed from acceleration and deceleration of moving observer. In relativistic environment, changing the speed's value will also change the Lorentz Factor value thus directly affect the Time Dilation value. One can simply calculate the dilated time by using Time Dilation equation, however if the velocity is not constant (from implying Special Relativity), one must repeatedly calculate the dilated time considering the changing value of displacement/distance and time. This paper discusses the proposed method by dissecting Time Dilation equation and relates it to computational method available nowadays in order to dynamically calculate and ultimately simulate Time Dilation effect in relativistic virtual environment.
\end{abstract}

Index Terms - Time dilation, simulation, special relativity.

\section{INTRODUCTION}

An intuitive understanding of Time Dilation effect is hard to develop. One of the problems with current available simulations is that it is not dynamic. Dynamic in this context is defined by the ability to persistently simulate Time Dilation effect in real-time interactive simulation. Real-time interactive simulation is an environment setting whereby user is able to control the input parameter thus simultaneously changing the output simulation in real-time.

This paper will start off with literature on Special Relativity Simulation that focuses on Time Dilation component within the simulation. Fundamental theory of Time Dilation will be discussed next together with its relation to Lorentz Factor specification which is the basis of Special Relativity postulation. The next section will be a detail discussion on a proposed method of how to dynamically simulate Time Dilation in relativistic environment where Length Contraction also being applied to the surrounding space. Time Dilation basic equation will be dissected and several parameters will be modified in order to become relevant with Frame per Second architecture of modern computer nowadays. The discussion will be concluded with a

Manuscript received November 28, 2014; revised May 22, 2015. This work was supported in part by the Department of Higher Education, Ministry of Education, Malaysia under the Fundamental Research Grant Scheme (FRGS), RDU130120 through Universiti Malaysia Pahang.

The authors are with the Faculty of Computer Systems \& Software Engineering, Universiti Malaysia Pahang, Malaysia (e-mail: abbas@ump.edu.my, zaifa@ump.edu.my, loklehleong@gmail.com). table showing all related activities needed to produce the objective simulation which is a dynamic Time Dilation effect in relativistic virtual environment.

\section{LITERATURE ON TIME DILATION SIMULATION}

A lot of effort to visualize Special Relativity using graphical computerize simulation have been done throughout recent years [1]-[7]. Most of them focused on visualizing relativistic environment from the effect of Lorentz Transformation. Although it is rare, there is also an attempt to simulate Lorentz Transformation effect in "real world" setting by warping real world camera captured images [8]. Time Dilation however did not get much attention. Even though some simulations include Time Dilation, it is just to visualize the general principle of dilated time over real-world time. Time dilation equation is just used to calculate the total dilated time taken by moving from point $\mathrm{A}$ to point $\mathrm{B}$ when the speed is near or equal to the speed of light.

Carr and Bossomaier attempted to visualize Time Dilation in their 2D game entitled "Relativity in a rock field". Dilated Time in this game is shown by the countdown timers on a special object that changed relative to the player's speed. This attempt is identified to be not dynamic because the dilated time is only relative to speed, not to time and space which define the value of velocity [1]. Ceperly's Special Relativity-Time Dilation attempt is merely just a 2D simulation to visualize the general concept of Time Dilation with the animation of The Light Clock experiment [2], thus also did not dynamically simulate the Time Dilation effect. Another attempt is by McGrath et al. [5] who simulated a time difference that changed depending on the location of the observer. The location however, is pre-defined by the simulation thus making the dilated time not relative to velocity of a moving observer.

Perhaps the most advanced Special Relativity simulation to date is a game named "A slower speed of light" by MIT Game Lab [4], [9]. Simulation of Time Dilation in this game however is only shown at the end of gameplay where the cumulative time taken to complete the given mission (with observer's speed is equal to the speed of light) is being compared to real world time therefore not dynamic in the sense that it does not persistently simulate Time Dilation as the player accelerate and decelerate in the game environment.

\section{TIME DILATION}

Time Dilation theory was proposed by Albert Einstein in his publication on Special Theory of Relativity [10]. Time Dilation theory indicates that time move slower when 
travelling near the speed of light. This phenomenon happens because the speed of light is defined as constant and because of this, time and space must change in order to become relative with the light's speed (relativity environment). The changing nature of space relative to light's speed is defined by Lorentz Transformation [11] and subsequently Length Contraction theory [12].

Following equation (1) shows the Time Dilation equation. This equation expresses the postulation that moving observer will experience slower time than stationary observer based on The Light Clock experiment. Using this equation, dilated time can be calculated based on constancy of the speed of light.

$$
\Delta t=\frac{\Delta \tau}{\sqrt{1-\frac{v^{2}}{c^{2}}}}
$$

Equation (2) shows the Lorentz Factor equation that is associated with Time Dilation equation. The association can be viewed in equation (3). Lorentz Factor is implemented in several equations in Special Relativity because it defines the changes of time, length and mass in relativistic environment.

$$
\begin{gathered}
\gamma=\frac{1}{\sqrt{1-\frac{v^{2}}{c^{2}}}}=\frac{1}{\sqrt{1-\beta^{2}}}=\frac{d t}{d \tau} \\
\Delta t=\gamma \Delta \tau=\frac{\Delta \tau}{\sqrt{1-\frac{v^{2}}{c^{2}}}}
\end{gathered}
$$

Based on the given equations and many related others [13], Time Dilation effect in relativistic virtual environment can possibly be simulated. It is however to consider that to simulate dynamic Time Dilation in moving observer's frame, both stationary and moving frames need to be merged. This is to enable the viewer to visualize the Time Dilation effect viewed by stationary frame while also visualize the Lorentz Transformation effect of the surround space viewed by moving frame.

\section{Dynamic Time Dilation Simulation}

Referring to the literature of graphical computerize simulation of Special Relativity, most of them are intended to be interactive in the sense that they became a game. This is not surprising given the evidence that game is a powerful tool to leave impacts on visual attention [14]. To quote Philip Tan, a Creative Director at MIT Game Lab, “The MIT Game Lab is built around this idea that play is extremely powerful, and one thing games are good at is giving people an intuitive grasp of complex scientific ideas [9].” As such, the implementation of visualizing dynamic Time Dilation effect in relativistic environment is seen best suited to be implemented using game platform/medium.

To persistently simulate Time Dilation effect in real-time interactive simulation, dilated time needs to be continuously calculated. As graphical computerize game tend to run in FPS
(Frame per Second) timeline, dilated time can be calculated continuously in every rendered frame. However, it needs to structurally consider which parameter to be calculated first because each parameter is required to calculate dilated time, and is relative to each other due to the nature of Special Relativity environment.

To further elaborate the possibility of dynamically simulate Time Dilation effect in relativistic virtual environment, Time Dilation equation as in (1) will be dissected and each essential parameters will be discussed. The $c$ parameter or the value of speed of light is the only constant parameter in relativistic environment with the value of $299792258 \mathrm{~m} / \mathrm{s}$. As the value of $c$ cannot be changed, it will not be further discussed in this section.

\section{A. Proper Time $(\Delta \tau)$}

Proper time is defined as elapsed time between two relativistic events as measured by a clock that passes through both events (same clock). For simulation created by modern computer, using computer internal clock as a measurement instrument can be considered as the best practice. It is because every frame rendering process in the computer will refer to this one clock despite various frame rate speeds. Hence, whether it is a moving or stationary observer, because both frames are being simulated using the same system, the same computer internal clock can always be the absolute reference. Therefore, proper time can be calculated by subtracting current frame clock with previous frame clock measured by the same computer internal clock. In other words, time/duration is what it takes to render previous frame.

It is important to bear in mind that the computer clock is World Clock. With that being the case, every consequent frame after the second frame where the observer is in motion (stationary in the first frame and start moving in the second frame) needs to consider previous frame's calculated dilated time. This is because the Proper Time is measured by a relativistic clock, not the World Clock. Hence, for every moving observer's second frame and forward, Proper Time is defined as time/duration that it takes to render previous frame minus dilated time in previous frame. As for deceleration of motion, the next frame after observer is in complete stationary position where the consideration for previous frame's calculated dilated time needs to be stopped (current frame will consider previous frame's dilated time while next frame will consider current frame's dilated time which is none because the observer is already in complete stationary position).

\section{B. Relative Velocity (v)}

In classical mechanics, velocity is defined as the rate of change of position (displacement in certain direction) with respect to time (4). It is also known that speed is the magnitude of velocity and in relativistic environment where speed can be equal to the speed of light, the displacement of certain object is highly dependent on Length Contraction phenomenon as defined in equation (5).

$$
v=\frac{d x}{d t}
$$




$$
L=\frac{L_{0}}{\gamma(v)}=L_{0} \sqrt{1-\frac{v^{2}}{c^{2}}}
$$

To simulate Special Relativity in real-time interactive simulation, moving observer needs to be able to accelerate and decelerate. And because both actions require variation of speed value during motion, the Length Contraction value will also be diversified. As such, to get a proper displacement value, Length Contraction needs to be first calculated before determining the displacement of the moving observer.

Open Relativity is an open-source toolkit (developed by MIT Game Lab) to simulate effects of Lorentz Transformation (including Length Contraction) by manipulating the value of the speed of light [15]. As such, by implementing Open Relativity in developed simulation/game, the displacement of an object can be directly calculated based on previous and current position of the object towards Cartesian Coordinate system using Pythagorean Theorem as in (6). This is because Length
Contraction phenomenon has already been taken into account by Open Relativity. Nevertheless, the calculation to get the displacement value of $d x$ needs to be done after Length Contraction is performed; as well as in every frame within FPS timeline to comply with other parameter's calculation time.

$$
c^{2}=a^{2}+b^{2}
$$

Referring back to velocity equation stated in (4), $d t$ value can be defined as elapsed time or duration it takes for $d x$ to be completed. As discussed earlier in Proper Time section, because computerized simulation run in FPS, time/duration to render previous frame can be used as elapsed time and because Proper Time already takes into account the dilated time from previous frame, it can also be used as the value for $d t$.

\begin{tabular}{|c|c|c|c|}
\hline Observer's position & Stationary & Stationary & Moving \\
\hline User action & None & Accelerate/Decelerate & Accelerate / Decelerate \\
\hline $\begin{array}{l}\text { Difference between World and } \\
\text { Relativistic Clock }\end{array}$ & None & None & None for first frame and Yes for consequent frame (Time Dilation) \\
\hline Parameter calculation & None & Speed & $\begin{array}{l}\text { Proper Time, Length Contraction, Displacement, Relative Velocity, Time } \\
\text { Dilation }\end{array}$ \\
\hline Effect to observer and/or space & None & Observer start moving & Length Contraction, Time Dilation \\
\hline
\end{tabular}

TABLE I: ACTIVITIES/ACTIONS IN DESIGNATED FRAMES WITHIN THE FPS TIMELINE

\section{Dynamic Dilated Time on Moving Observer}

Based on discussed parameters in previous sections and Time Dilation equation stated in (1), several parameters can be modified to obtain the value of dilated time in moving observer's frame. The modified parameters in respect to Time Dilation equation is defined in (7).

$$
\Delta t_{2}=\frac{\Delta \tau-\Delta t_{1}}{\sqrt{1-\frac{\left(\frac{d x}{\Delta \tau-\Delta t_{1}}\right)^{2}}{c^{2}}}}
$$

Equation (7) shows the modified parameters for Proper Time $\Delta \tau$ and Relative Velocity $v$ where both parameters are associated with Dilated Time from previous rendered frame $\Delta t_{1}$. As discussed in previous Relative Velocity section of this paper, $d x$ is a displacement of observer with respect to Length Contraction of its surround space. Because Length Contraction phenomenon is highly dependent on the speed of the observer, and also because the speed is highly correlated with acceleration and deceleration state of the observer, further discussion in regards to the speed of the observer will be presented in the next section.

\section{Speed of the Observer}

To dynamically simulate Time Dilation effect in real-time interactive simulation, user of the simulation system needs to be able to control the speed of the observer. By enabling this interaction, user will be able to visualize Time Dilation effect as they accelerate and decelerate in the relativistic environment themselves.

Speed is defined by rate of motion or rate of change of position of the observer. In relativistic environment, the maximum value of velocity is $299792258 \mathrm{~m} / \mathrm{s}$ which is the value of the speed of light. Because the relativistic nature of space time can only be distinctively seen when speed is almost equal to the speed of light, the rate of acceleration and deceleration in the simulation need to be within a ratio in which the increase and decrease value of speed is significant. As an example, "A slower speed of light" game by MIT Game Lab uses a ratio of 100:1 where a 100 magical orbs needs to be collected by player/user (the observer) in order to equalize with the speed of light [4], [9]. By this ratio, it is assumed that every increment of speed (or in the game case is every decrement of the light's speed) is equal to 2997922.58 $\mathrm{m} / \mathrm{s}$ (the speed of light divided by 100 ).

As for the dynamic simulation where speed value is diversify due to acceleration and deceleration motion of the observer, the ratio of speed towards the speed of light can also be 100:1 where maximum speed is equal to the speed of light. As such, every user's action of accelerate and decelerate (by giving certain input such as a button pressed) are equal to increase or decrease value of $2997922.58 \mathrm{~m} / \mathrm{s}$ respectively. Despite other parameters calculation are required in every frame within the FPS timeline, speed parameter will only be calculated if action of motion (accelerate or decelerate) from the user is detected.

\section{E. Activities/Actions within Designated Frames}

Previous sections have discussed ways in which each parameter within Time Dilation equation can be calculated in 
order to get the needed value. As mentioned before, each calculation will be processed in designated frames within the FPS timeline in specific sequence that depends on the state of the observer whether it is stationary or moving (accelerate and decelerate). The following Table I illustrates activities/actions in regards to observer's position that relates to user's input, difference between World Clock and Relativistic Clock, all required parameters calculation and effect of all activities/actions toward observer and/or its surrounding space.

\section{CONCLUSION}

This paper critically reviews several Special Relativity simulation applications and proposes a method to dynamically simulate Time Dilation effect. The method is proposed by dissecting Time Dilation equation and suggests a method to calculate each required parameters within processed frames in FPS timeline. It is important to bear in mind that to completely visualize Time Dilation effect in relativistic environment of moving observer's frame, both stationary (World Clock) and moving (Relativistic Clock) frames need to be merged. This is to enable the viewer to visualize the Time Dilation effect viewed by stationary frame (by comparing Relativistic Clock towards World Clock), while also visualize the Lorentz Transformation effect of the surround space viewed by moving frame.

\section{REFERENCES}

[1] D. Carr and T. Bossomaier, "Relativity in a rock field: A study of physics learning with a computer game," Australasian Journal of Educational Technology, vol. 27, no. 6, pp. 1042-1067, 2011.

[2] P. Ceperley. (2011). Special relativity-time dilation. [Online]. Available: http://digilib.gmu.edu/dspace/handle/1920/6569

[3] T. Doat, E. Parizot, and J. M. Vezien, "A carom billiard to understand special relativity," in Proc. IEEE Virtual Reality Conference (VR), 2011, pp. 201-202.

[4] K. Gerd, T. Philip, and S. Steven, "Slower speed of light: Developing intuition about special relativity with games," in Proc. the International Conference on the Foundations of Digital Games, New York, USA, 2013, pp. 400-402.

[5] D. McGrath, C. Savage, M. Williamson, M. Wegener, and T. McIntyre, "Teaching special relativity using virtual reality," in Proc. The Australian Conference on Science and Mathematics Education, 2012.
[6] M. Wegener, T. J. McIntyre, D. McGrath, C. M. Savage, and M. Williamson, "Developing a virtual physics world," Australasian Journal of Educational Technology, vol. 28, no. 3, pp. 504-521, 2012.

[7] D. Weiskopf, "A survey of visualization methods for special relativity,” Scientific Visualization: Advanced Concepts, pp. 289-302, 2010.

[8] D. Weiskopf, D. Kobras, and H. Ruder, "Real-world relativity: Image-based special relativistic visualization," in Proc. the Conference on Visualization '00, 2000, pp. 303-310.

[9] E. Rik. (2013). Playing with Einstein. [Online]. Available: http://web.mit.edu/newsoffice/2013/playing-with- einstein.html

[10] A. Einstein, "On the electrodynamics of moving bodies," Annalen der Physik, vol. 17, no. 891, p. 50, 1905.

[11] W. Engelhardt, "On the origin of the Lorentz transformation," arXiv preprint arXiv: 1303.5309, 2013.

[12] F. Smarandache, "Oblique-length Contraction factor in the special theory of relativity," Progress in Physics, vol. 1, pp. 60-62, 2013.

[13] D. C. Cassidy, G. J. Holton, and F. J. Rutherford, Understanding Physics, New York: Springer, 2002.

[14] D. B. Vallett, R. L. Lamb, and L. A. Annetta, "The gorilla in the room: The impacts of video-game play on visual attention," Computers in Human Behavior, vol. 29, no. 6, pp. 2183-2187, 2013.

[15] K. Gerd, T. Philip, S. Zach, C. Ryan, S. Steven, and S. Sonny. (2013). Open relativity. [Online]. Available: http://gamelab.mit.edu/research/openrelativity/

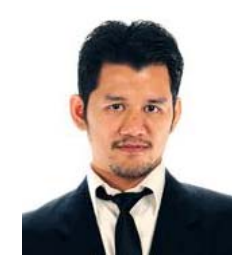

Abbas Saliimi Lokman was born in Kelantan, Malaysia on January 13, 1984. He received his bachelor degree of computer science (software engineering) in 2007 and the master degree of computer science in 2011 with a thesis entitled "Chatbot Algorithms in Data Representation for Diabetes Education” from Universiti Malaysia Pahang, Malaysia.

Currently, he is with Faculty of Computer Systems \& Software Engineering, Universiti Malaysia Pahang, Malaysia with a handful responsibility as a full-time lecturer. His last publication is "Enhancement algorithms for SQL-based Chatbot" that were published in Springer's Communications in Computer and Information Science (CCIS), vol. 180, part 5, pp. 470-479, 2011. His latest Google Scholar h-index since 2009 is 2.

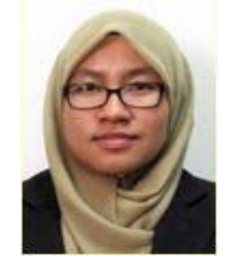

Ngahzaifa Ab. Ghani was born in Seremban, Negeri Sembilan on January 9, 1983. She received her first bachelor degree of science (geonformatic) from Universiti Teknologi Malaysia in 2006. In 2012, she received her $\mathrm{PhD}$ degree from The University of Nottingham, UK.

Currently, she is with Universiti Malaysia Pahang. Her research interests include data mining, machine learning and geospatial science. 